\title{
Sensor Network Based Automatically Opertable Barricadeson Public Railway Crossing
}

\author{
Shivani Madhok, Udita Jindal, Shriya Chauhan \\ Chitkara University Research and Innovation Network \\ Chitkara University \\ Chandigarh, Punjab \\ shivanimadhok0@gmail.com, uditajinda194@yahoo.in, \\ shriya2194chauhan@gmail.com
}

Abstract - Railway safety is a crucial aspect of rail operation across the globe. In our country, there are no strong steps are taken against the unmanned level crossings and due to this accidents are increasing day by day. There is a huge increase in number of accidents as compared to last decade. India's railway system is the biggest railway network all over the Asia. And this huge network cannot be handled manually. To manage each and every railway crossing manually is not possible. Our paper basically deals with a prototype model of automatic railway barricades and discussed its working and construction. To avoid these accidents and to save road users time we have designed it. In this research work, we have placed two sensors near to the gate and these sensors will detect the arrival of the train and road users will be informed using traffic lights about the status of the train. This whole operation is automatic there is no need of human powers and is less risky and less time consuming. This automatically operatable railway barricades has increased safety of users. There are many countries in which this automatic gate control system has been implemented. In Japan, it is labeled as Automatic Train Control (ATC). Other countries with successful implementations of this system are South Korea,United Kingdom,United States, Africa etc.

\section{Keywords-Railway Gate, Level Crossing, Automatic Train Control}

\section{INTRODUCTION}

Indian railway network is the biggest rail network in the Asia. It is impossible to supervise each and every rail crossing so there are so many unattended railway crossings this case is more often seen in rural and remote areas. This huge railway network cannot be handled or managed manually. Due to this lack of supervision there are numbers of accidents that take place all over the India. The Times of India dated July 25, 2014 published an article about railway accidents and wrote there are 30,348 level crossings in India, of which around $40 \%(11,563)$ are unmanned. In last five years, 723 people were killed in accidents at level crossings. According to annual report, accidents at Level Crossings accounting for $22 \%$ of the total accidents on Indian Railway were responsible for $49 \%$ of total fatalities during the last decade. There is increase in number of accidents year by year. Railroad related accidents are more dangerous than other on road accidents in terms of death rate. Therefore more efforts are needed for improving safety[1].This paper is to provide an automatic railway gate at a crossing replacing the gates that are operated by the gatekeeper. The road users 
have to wait long for the gatekeeper to come and manually open the barricade this is so time consuming and this is also a major factor which leads to the need of automatic system. This paper is basically stressed to avoid railway accidents happening at unattended railway gates.To avoid the expense resulting from railway crossing accidents, it would be highly desirable if a crossing gate is automatic, this will prove to be practically indestructible [2]. Figure 1 represents the working or work flow of the automatic operatable barricades that how the comparator IC and motor driving IC reacts to the IR module. Figure 2 represents the components used in the circuit and main components used are IR module, L293D, LM358.

Figure 1: Work Flow of Automatic Operatable Barricades

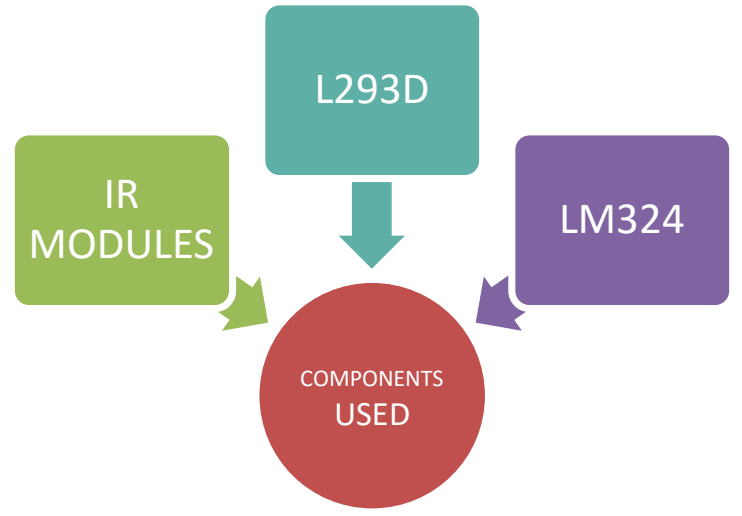

Figure 2: Components Used In Automatic Operatable Barricades

\section{Automaticrailway}

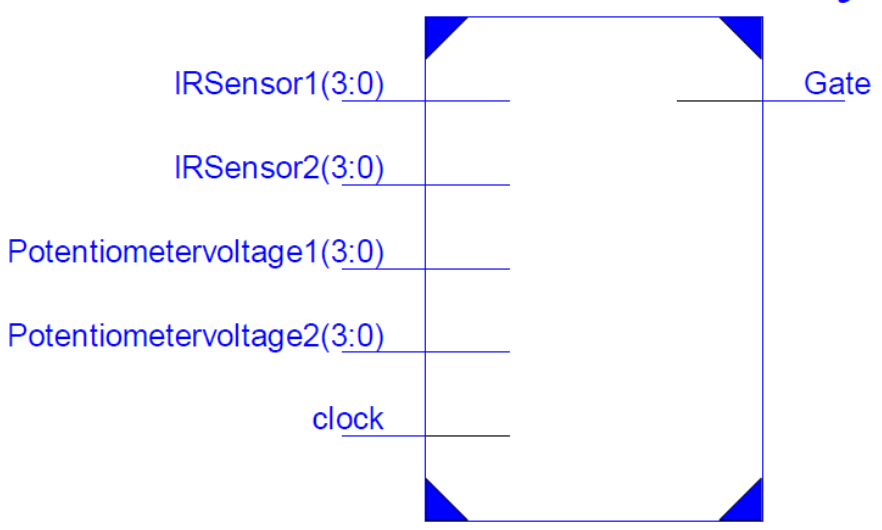

Figure 3: Symbol for Automatic Railway Barricades 
Figure 3 represents symbol of automatic railway barricades. From its synthesis report we came across different results such as:

Automatic FSM Extraction: YES

FSM Encoding Algorithm: Auto

Safe Implementation: No

FSM Style: LUT

RAM Extraction: Yes

RAM Style: Auto

ROM Extraction: Yes

Shift Register Extraction: YES

ROM Style: Auto

Resource Sharing: YES

Asynchronous To Synchronous: NO

Shift Register Minimum Size: 2

Use Clock Enable: Auto

Use Synchronous Set: Auto

Use Synchronous Reset: Auto

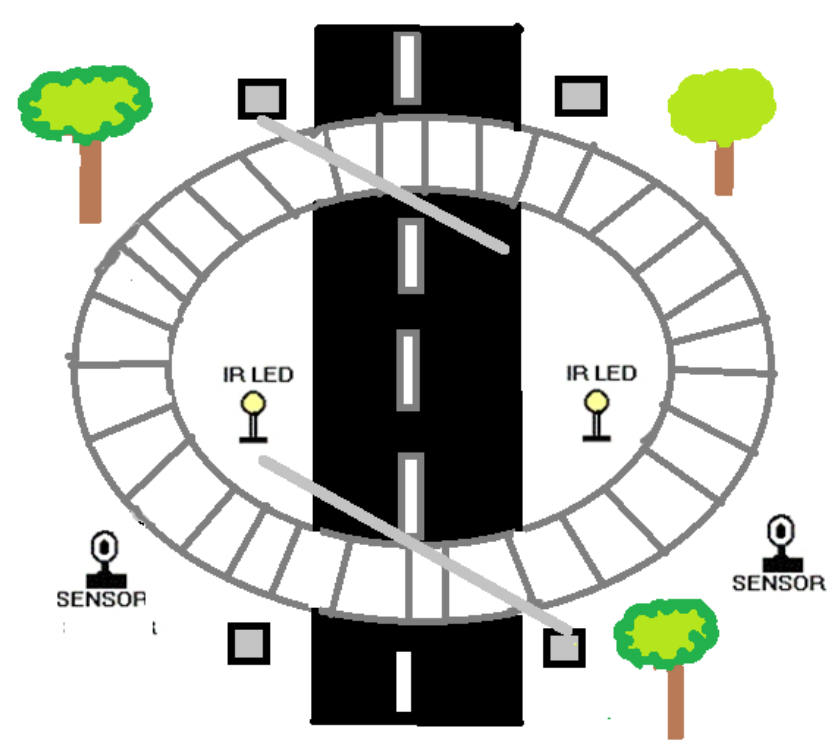

Figure 4: Railway Track Layout

We have made a prototype of automatic railway gate control in which we have used IR (infrared) modules as sensors. The presence of the train is detected by the sensors. If train is approaching near the railway crossing the barriers shuts down and once the train has passed away barriers opens by itself. On both sides of the barrier these modules are kept and when the train cuts the $1^{\text {st }}$ IR module the barriers should close and when train reaches the $2^{\text {nd }}$ module the barriers should open it. Arrangement of the IR modules can be seen in figure no. 4. Instead of using a single module we have used modules in AND logic. When all the IRs are cut then only operation is performed. This is done to avoid the confusion weather it is a train or any other animal, human being. Gate includes operational mechanism which moves a gate between a closed position and an open position. [2].Inthis project we detect the arrival of train and warn the road users about the arrival of the train using traffic signals. 2 LEDs are connected with the IR modules 
when the train cuts $1^{\text {st }}$ module red LED lights up and when $2^{\text {nd }}$ module is cut green LED comes in its role.In this way the road users come to know about the status of the train. When foreside sensor gets activated the sensed signal is sent to the comparator and the gate is closed and stays closed until the train crosses the gate and reaches the other side sensors. When the other side sensors are activated signal about the departure is sent to the comparator and motor turns in opposite direction and gate opens and motor stops automatically [1]. Next thing that we have used is LM324 IC as a comparator IC. In reference [5], LM324 is used to convert heart bit into electrical signal. LM324 is a fourintegration operation amplifier. Due to its low cost and easy to use, it is widely used in control and magnification of general signals [6-8]. Here, this comparator will compare the values one from the IR module and other from the potentiometer. The potentiometer is used for comparing the values in comparator.Potentiometer of 10 Kilo Ohms is used. Figure 3 shows the pin diagram of LM324.

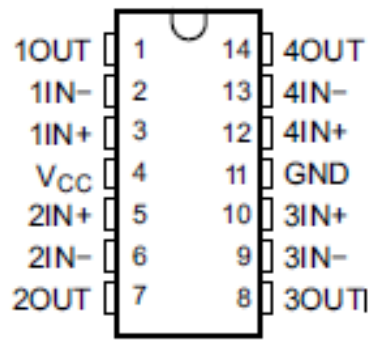

Figure 5:Pin Diagram of LM324

Now, next is our motor driving IC (L293D) this IC will drive our motor as we want to in clockwise or in anticlockwise direction. Figure 4 shows the pin diagram of L293D.In this IC 1Y, 2Y, 3Y, 4Y are the pins where motor is to be attached and 1A, 2A, 3A, 4A are the input pins from the other circuit. In reference [9], channels are used for speed controlling and direction controlling along with a motor driver IC L293D.

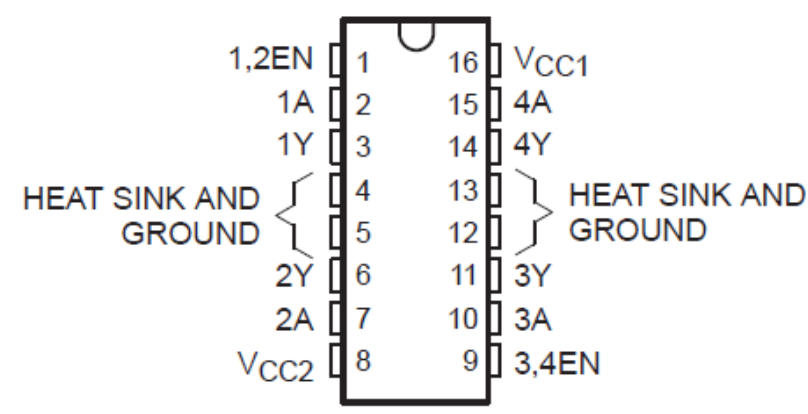

Figure 6:Pin diagram of L293D

Pin diagram of L293D is shown in Figure 6. Figure 4, 6 represents project display, sensor placement, and use of IR LED in automatic railway crossing barriers. 


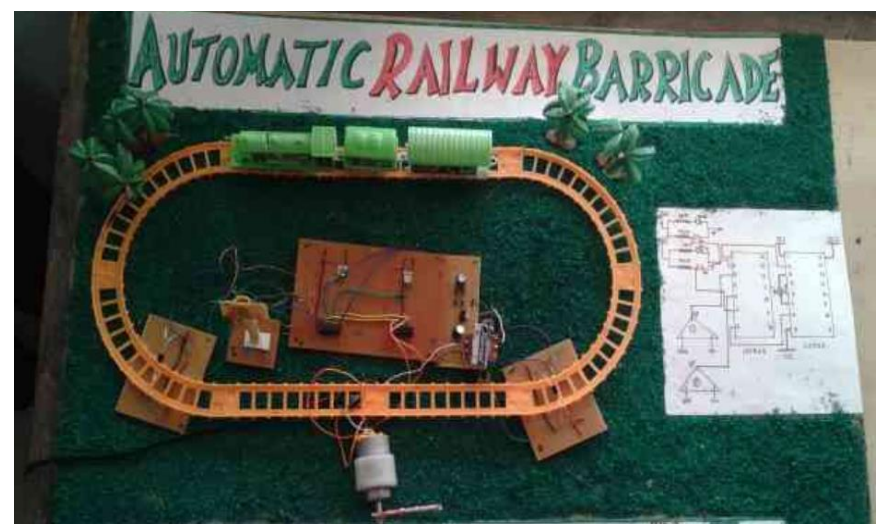

Figure 7:Automatic Railway Barricade Model

\section{CONCLUSION}

Automatically OpertableLevel Crossings on Public Railway Crossing is a system which does not require any human resource to be present. Each and every action is performed with the help of motors and there is no usage of human powers. This system also reduces the chances of human errors. This whole system is operated automatically with the help of sensors.In this research work, we have used IR sensors and placed them at a distance from each other. The sensors are arranged in AND logic to avoid confusion between train and other things. It is found to be stable and very reliable. Thecircuit is able to control the railway gate. The circuit is tested properly and is working in both directions clockwise and anticlockwise. This model has reduced human powers and is much safer as compared to manual gatekeepers and the road user does not have to wait long for the gatekeeper to open the barriers. The whole operation is automatic and can be controlled by just sitting in a control room and supervising the actions.

\section{FUTURE SCOPE}

This is a very basic and general working model and a lot more can be done in it. Indian Railway is suffering from level crossing accidents so this project can be presented in front of them. This is a low level project but it can be implemented at a larger scale and secure many lives.This model is focused on automatic railway barricades using IR sensors instead of these IR sensors we can use proximity sensors. To detect the train from a distance we can use ultrasonic sensors which will display the distance how far is the train from the sensors and it can be displayed on LCD. This model has no limit it can be extended as per the requirements we can make an Anti-Collision system in which we can attach an IR LED in front of each and every engine. Whenever the two trains coming closer face to face these IR will detect them and we can program it either to change the tracks if possible or stop. This model is automatic but it can be made wireless and can be operated remotely by using an Android Device through Android Applications by the station master. GSM, Bluetooth and RF can also be used for wireless communication. Advancement in this research work also includes speed limiter a sensor can be placed to detect the speed of the train and a limit is set if the train exceeds that particular limit brakes should come into action and slower down the speed of the train. Microcontrollers can also be used. Micro controller (AT89C51) allows dynamic and faster control.

\section{REFERENCES}


[1]. A. Zuhairi, A. S. Mahdi. "Automatic Railway Gate and Crossing Control based Sensors \& Microcontroller.", International Journal of Computer Trends and Technology(IJCTT), volume 4, Issue 7, July 2013.

[2]. T.A. Barvinek, and J. W. Jensen. "Railroad crossing gate." U.S. Patent No. 4,897,960. 6 Feb. 1990.

[3]. C. W. E.Cobb,. "Automatically operable automotive vehicle gate apparatus provided with self protection and automotive protection." U.S. Patent No. 4,364,200. 21 Dec. 1982.

[4]. J.Banuchandar, et al. "Automated Unmanned Railway Level Crossing System." International Journal of Modern Engineering Research (IJMER) 2.1 (2012): 458-463.

[5]. R. Sukanesh, et al. "Cellular phone based biomedical system for health care." Communication Control and Computing Technologies (ICCCCT), 2010 IEEE International Conference on. IEEE, 2010.

[6]. T. A. N.Jian-jun, "A High Performance and Low-cost Instrumental Amplifier Based on LM324 [J]." Telecommunication Engineering 3 (2004): 038.

[7]. I. Lopez-Calle, et al. "Two-Photon Absorption (TPA) backside pulsed laser tests in the LM324." Radiation and Its Effects on Components and Systems (RADECS), 2009 European Conference on. IEEE, 2009.

[8]. W. A. N. G. Z. Yong, and W. E. N. Guo-dian. "Design and Simulation of Signal Generator Based on LM324 [J]." Modern Electronics Technique 12 (2011).

[9]. S. R. Khan, and M. S. Bhat. "GUI based industrial monitoring and control system." Power and Energy Systems Conference: Towards Sustainable Energy, 2014. IEEE, 2014. 